\title{
PREVALENCE OF RADIOLOGICALLY DEFINED OSTEOARTHRITIS IN THE FINGER AND WRIST JOINTS OF ADULT RESIDENTS OF TECUMSEH, MICHIGAN, 1962-65
}

\author{
William J. Butler,${ }^{1 *}$ Victor M. Hawthorne, ${ }^{2} \dagger$ William M. MikKelsen, ${ }^{3}$ \\ Wendy J. Carman, ${ }^{2}$ Donald L. Bouthillier, ${ }^{2}$ Donald E. Lamphiear ${ }^{2}$ and \\ IMTIAZ U. KAZI
}

Departments of ${ }^{1}$ Biostatistics and ${ }^{2}$ Epidemiology, School of Public Health and ${ }^{3}$ Department of Internal Medicine, School of Medicine, University of Michigan, Ann Arbor, MI 48109, U.S.A.

(Received in revised form 2 October 1987)

\begin{abstract}
Radiographs of the fingers and wrists of adult participants in the Tecumseh Community Health Study in 1962-65 were examined for signs of osteoarthritis (OA). The severity of OA for each of 32 joints of the fingers and wrists was recorded for each individual. Attention was restricted to the 3035 participants who were 32 years of age or older and for whom a diagnosis of OA was available for each of 32 joints. Joint-specific prevalence rates of OA increased sharply with age for both sexes, and at the older ages, the prevalence rates for most joints were higher for females. Older individuals with $O A$ also had a greater number of affected joints, with females having a greater number of affected joints than males. Of those individuals aged 44 years or younger, only $6.2 \%$ had one or more joints affected with $\mathrm{OA}$. The percentages were 21.6 and $42.0 \%$ for those aged $45-59$ years and 60 or more years, respectively. The distal interphalangeal (DIP) joints were the most frequently affected joints in all age categories for both sexes and OA in the proximal interphalangeal (PIP) joints was positively associated with OA in the DIP joints. However, controlling for the number of affected DIP joints, the PIP joints of older subjects were more likely to exhibit OA than the PIP joints of younger subjects. Though there is an association between OA in the DIP and PIP joints, there was only a small, nonsignificant association $(\mathrm{OR}=1.24,95 \% \mathrm{CI}=0.83,1.84)$ between disease in the DIP and PIP joints of the same finger.
\end{abstract}

Osteoarthritis Epidemiology Prevalence

\section{INTRODUCTION}

Disease of the musculoskeletal system is among the most common afflictions of the human race and osteoarthritis $(\mathrm{OA})$ is the most frequently occurring joint disease [1]. Osteoarthritis, also called degenerative joint disease, occurs throughout the body but the joints of the hands, knees, and feet are the most commonly affected, along with the spine, hips, sacroiliac, and tarsi [2]. Prevalence of $\mathrm{OA}$ in the hands and wrists increases sharply with age. The Health Exam-

*Current address: Failure Analysis Associates, 2225 East Bayshore Road, Palo Alto, CA 94303, U.S.A. †Author to whom reprint requests should be addressed. WDeceased. ination Survey of the U.S. in the 1959-1962 found that the prevalence of radiologically defined $O A$ in the hands and wrists increased from $4.8 \%$ in those aged $35-44$ years to $78.7 \%$ in those aged 75-79 years [3, Table 2]. Similar prevalence rates for the U.S. and Western Europe have been reported by other researchers [4, Table 1]. In 1960-62, 40.5 million Americans were estimated to suffer from OA [3], but the number currently exhibiting the disease would be higher because of the shift in the population to the older age categories.

Osteoarthritis in specific joints may result from acute trauma to the joint, such as fractures, dislocations, sprains, or other joint injury $[1,5]$. Long-term, low level trauma or stress may 
also explain some of the occurrence of disease in multiple joints $[6,7]$. Individuals engaged in certain occupational and recreational activities develop OA more frequently in those joints stressed by their activities $[1$, p. $84 ; 6]$, though exceptions have been observed $[1$, p. 84]. Systemic and constitutional factors have also been correlated with the disease [8], including serum uric acid [9] and obesity [2, 9, 10]. Importantly, obesity is associated with $\mathrm{OA}$ in nonweightbearing joints such as the hands and wrists. Genetic factors associated with Heberden nodes are associated with both the occurrence and severity of the disease [5]. A substantial proportion of the incidence of $\mathrm{OA}$ is unexplained by these few risk factors, however, and the pathogenesis of the disease remains unknown [11]

In this report 32 joints in the fingers and wrists are scored for $\mathrm{OA}$, and the pattern of disease is described. Attention is focused on examining how this pattern differs among age and sex groups and whether there is an association in disease status between joints on the same finger. A previous report on this population [12] did not record findings for each joint separately but instead reported for each individual only the highest score of OA exhibited by any of the joints. This was consistent with much of the epidemiologic work on OA at that time, but substantial information on the patterrn and severity of the disease was not utilized.

\section{METHODS}

\section{Data collection}

A comprehensive longitudinal epidemiologic investigation of the entire population of Tecumseh, Michigan and environs was begun in 1956. The broad objective of the project was to study the natural history of chronic disease. Beginning in 1959 participants in the Tecumseh Community Health Study have been surveyed at several intervals using both trained lay interviewers and self-administered questionnaires. Physicians from the University of Michigan reviewed questionnaires, collected additional medical information and did physical examinations at each of the first three cycles of examinations. Participation rates for the baseline examinations were always higher than $80 \%$ [13].

During the second cycle of examinations in 1962-1965, X-rays of the hands and wrists were obtained for $96 \%$ of the participants age 20 years or older [12]. A single radiograph of the postero-anterior view of both hands and wrists was taken, employing an average exposure of $0.3 \mathrm{~s}$ at $100 \mathrm{~mA}$ and $46 \mathrm{kVp}$. Attention is restricted in this report to 3163 subjects who were $X$-rayed in 1962-65 and who were aged 32 and older at the time of their X-rays.

The degree of $\mathrm{OA}$ in individual joints is scored on a five point scale $(0=$ none, $1=$ minimal, $2=$ mild, $3=$ moderate, $4=$ severe) according to an internationally accepted standard [14]. The degree of OA for each of the 32 joints for each individual was scored by a single reader (IK) without knowledge of the diagnoses made at the time of the previous report on this population [12]. Diagnosis of OA for all the joints was possible for 3035 subjects $(96 \%)$. This is the group examined in this report.

\section{Reliability of scores for $O A$}

The Atlas of Standard Radiographs does not provide a precise definition of the various grades for radiological diagnosis of OA [14]. It presents primarily a visual definition based on reproductions of radiographs of $\mathrm{OA}$ of varying severity in the individual joints. The accompanying verbal definitions are very sketchy, lack precision and clarity, and are not uniform for all joints. Thus, the grading of OA can be affected substantially by subjective judgment, which in turn may influence the conclusions of a research project $[1,15]$. Since a "Gold standard" for the diagnosis of $\mathrm{OA}$ is not available, the best that can be expected is that a reader be consistent with himself and with existing standards [14].

The reliability of the scores of OA used in this research was examined in three ways. First, the $\mathrm{X}$-rays of the 4943 joints from 155 individuals (selected as every twentieth X-ray) were scored by a second reader (WMM) so as to assure continued adherence to the standard. The agreement between the two readers on the scores for the individual joints was very high (weighted kappa $=0.73$ [16, Chapter 13]). The scores assigned by the two readers never differed by more than a single diagnostic category (for example, mild vs moderate). Because of the subjective nature of the diagnosis of $\mathrm{OA}$, these small differences could not be completely resolved and the diagnosis of the major reader (IK) was used. The purpose of this double reading was to avoid a "drift" in scoring by the major reader (IK) [15].

Secondly, the reliability of the scoring of the major reader (IK) was examined by comparing two separate readings by him of the $\mathrm{X}$-rays for 
Table 1. Disagreement in diagnosis of osteoarthritis in each of 32 joints of the hand and wrist, between first and second reading of X-rays, Tecumseh, Michigan, 1962-65

\begin{tabular}{|c|c|c|c|c|c|c|c|c|c|}
\hline \multirow{2}{*}{\multicolumn{2}{|c|}{ First reading }} & \multicolumn{8}{|c|}{ Second reading } \\
\hline & & \multicolumn{4}{|c|}{$\begin{array}{l}\text { Disagree by one } \\
\text { category }\end{array}$} & \multicolumn{4}{|c|}{$\begin{array}{l}\text { Disagree by two or } \\
\text { more categories }\end{array}$} \\
\hline Grade & joints & Higher & Lower & No. & $\%$ & Higher & Lower & No. & $\%$ \\
\hline 0 & 26,682 & 293 & - & 293 & 1.1 & 5 & - & 5 & $<0.1$ \\
\hline 1 & 1,059 & 21 & 523 & 544 & 51.4 & 0 & - & 0 & 0 \\
\hline 2 & 171 & 1 & 91 & 92 & 53.8 & 0 & 34 & 34 & 19.9 \\
\hline 3 & 15 & 2 & 5 & 7 & 46.7 & - & 5 & 5 & 33.3 \\
\hline 4 & 27 & - & 10 & 10 & 37.0 & - & 3 & 3 & 11.1 \\
\hline Total & 27,954 & 317 & 629 & 946 & 3.4 & 5 & 42 & 47 & 0.2 \\
\hline
\end{tabular}

876 individuals. These duplicate readings were performed at different points in time and the reader was not told that he was reading the same $\mathrm{X}$-ray for a second time. The reader was very reliable in assigning scores for OA to this group of test subjects (weighted kappa $=0.81$, [16, Chapter 13]). The agreement between the two readings for each joint for this test group is also presented in Table 1 using the same format as in the New Haven study [15]. Overall there was disagreement in only $3.6 \%$ of the joints, and the magnitude of disagreement increases with the severity of the disease. This amount of disagreement is consistent with that reported by Wright and Acheson [15] and indicates that the intra-reader variation in this study is consistent with that reported by others. Thirdly, the reader's (IK) scores were compared with the scores reported in the earlier report on this population [12]. Comparison could be made only on a summary basis for each individual using the score for the most severely affected joint. The current reader agreed exactly with the score reported in 1970 for $58 \%$ of the subjects and was within one category for $95 \%$ of the subjects. There was disagreement by two or more categories for $5 \%$ (155 out of 3035 ) of the subjects. However, the current reader diagnosed less severe OA for most of the joints where there was disagreement. Thus, the prevalence rates reported in this article are somewhat lower than those reported earlier [12].

\section{Statistical analysis}

In this analysis the five diagnosis categories for $O A$ are reduced to two categories: none, minimal vs mild, moderate, severe $[4,7]$. Standard chi-square tests and linear regression analysis are used in the analysis of these data. Statistical methods which treat subjects, instead of joints, as the units of observation were se- lected to avoid the complications introduced by the correlation among joints from the same individual. The sample size in each analysis is determined by the number of subjects involved, not the number of joints.

Mantel-Haenszel methods for matched analysis are used when the association between disease in specific joints of the same fingers is examined [16]. Methods for matched data are needed to accommodate the correlation among fingers from the same individual [16].

\section{RESULTS}

The prevalence rates for OA, averaged across the right and left hands, are presented in Table 2. These rates increase with age and are greater for females than for males for almost all joints in each age category. However, the observed differences between the sexes are not statistically significant for all joints in all age categories. Statistically significant differences are observed more frequently in the older age categories where there are higher joint-specific prevalence rates and greater differences in prevalence between the sexes.

Among younger subjects, practically all the $\mathrm{OA}$ is concentrated in the distal interphalangeal (DIP) joints of the four fingers. Among the older subjects, the DIP joints again have the highest prevalence rates but there is also disease in the proximal interphalangeal (PIP) and metacarpophalangeal (MCP) joints. The prevalence rates generally decrease along the length of each finger with the DIP joints having the highest rates and the MCP joints the lowest. Among the DIP joints, the highest rates were found in the index finger, consistent with the report by Acheson et al. [7]. The pattern in the thumbs differs from that of the fingers, with the highest rates in the first carpometacarpal joint (MCC). 
Table 2. Percentage* of individuals with osteoarthritis in each of the joints of the hands and wrists, Tecumseh, Michigan, $1962-65$

\begin{tabular}{|c|c|c|c|c|c|c|c|c|c|c|}
\hline \multirow[b]{2}{*}{ Age $\leqslant 44$} & \multicolumn{5}{|c|}{ Males $(n=1441)$} & \multicolumn{5}{|c|}{ Females $(n=1594)$} \\
\hline & Thumb & Index & Middle & Ring & Little & Thumb & Index & Middle & Ring & Little \\
\hline \multicolumn{11}{|l|}{ Joint $†$} \\
\hline DIP & & 1.0 & 0.4 & 0.4 & 1.1 & & $2.0 \ddagger$ & $1.8 \ddagger$ & 0.4 & 0.9 \\
\hline PIP & 0.1 & 0.1 & $0.4 \ddagger$ & 0.1 & 0.0 & 0.0 & $0.0^{\circ}$ & $0.0^{\circ}$ & 0.0 & 0.0 \\
\hline MCP & 0.1 & 0.0 & 0.0 & 0.0 & 0.0 & 0.0 & 0.0 & 0.0 & 0.0 & 0.0 \\
\hline $\mathrm{MCC}$ & 0.1 & & & & & 0.1 & & & & \\
\hline \multirow[t]{2}{*}{ Wrist } & \multicolumn{5}{|c|}{$0.5 \ddagger$} & \multicolumn{5}{|c|}{0.1} \\
\hline & \multicolumn{5}{|c|}{ Males $(n=494)$} & \multicolumn{5}{|c|}{ Females $(n=512)$} \\
\hline Age $45-59$ & Thumb & Index & Middle & Ring & Little & Thumb & Index & Middle & Ring & Little \\
\hline \multicolumn{11}{|l|}{ Joint } \\
\hline DIP & & 5.3 & 2.3 & 1.5 & 3.3 & & $9.3 \ddagger$ & $8.0 \ddagger$ & $3.3 \ddagger$ & $6.8 \ddagger$ \\
\hline PIP & 1.1 & 0.0 & 0.8 & 0.7 & 0.4 & 1.8 & $0.9 \ddagger$ & 1.4 & 0.6 & 1.1 \\
\hline MCP & 0.3 & 0.0 & 0.2 & 0.0 & 0.0 & 0.0 & 0.0 & 0.0 & 0.2 & 0.0 \\
\hline $\mathrm{MCC}$ & 1.4 & & & & & $2.6 \ddagger$ & & & & \\
\hline Wrist & \multicolumn{5}{|c|}{$0.6 \ddagger$} & \multicolumn{5}{|c|}{0.1} \\
\hline & \multicolumn{5}{|c|}{ Males $(n=250)$} & \multicolumn{5}{|c|}{ Females $(n=326)$} \\
\hline Age $60 \leqslant$ & Thumb & Index & Middle & Ring & Little & Thumb & Index & Middle & Ring & Little \\
\hline \multicolumn{11}{|l|}{ Joint $\dagger$} \\
\hline DIP & & 9.8 & 4.6 & 3.0 & 6.2 & & $21.8 \ddagger$ & $13.2 \ddagger$ & $7.9 \ddagger$ & $17.4 t$ \\
\hline PIP & 4.8 & 1.6 & 3.0 & 3.4 & 1.8 & 6.3 & $7.4 \ddagger$ & $10.2 \ddagger$ & $5.8 \ddagger$ & $5.7 \ddagger$ \\
\hline MCP & 0.2 & 3.0 & $3.4 \ddagger$ & 1.2 & 0.4 & 0.0 & 1.4 & 2.0 & 0.6 & 0.6 \\
\hline MCC & 6.0 & & & & & $13.5 t$ & \multirow{2}{*}{\multicolumn{4}{|c|}{0.8}} \\
\hline Wrist & \multicolumn{5}{|c|}{1.6} & & & & & \\
\hline
\end{tabular}

*The percentage for each joint is the average of the prevalences in the right and left hands.

$\uparrow \mathrm{DIP}$ - distal interphalangeal; PIP-proximal interphalangeal; $\mathrm{MCP}$-metacarpophalangeal; $\mathrm{MCC}$-carpometacarpal. $\ddagger p<0.05$ comparing males with females. No adjustments have been made for multiple comparisons.

This differs from the pattern in the thumb reported by Acheson et al. [7].

The age-sex specific prevalence rates of subjects with at least one joint affected with $\mathrm{OA}$ are displayed in Fig. 1. The prevalence rates for the youngest subjects are low and of about the same magnitude for males and females (6.6 and 5.8\%, respectively). The prevalence rates increase with

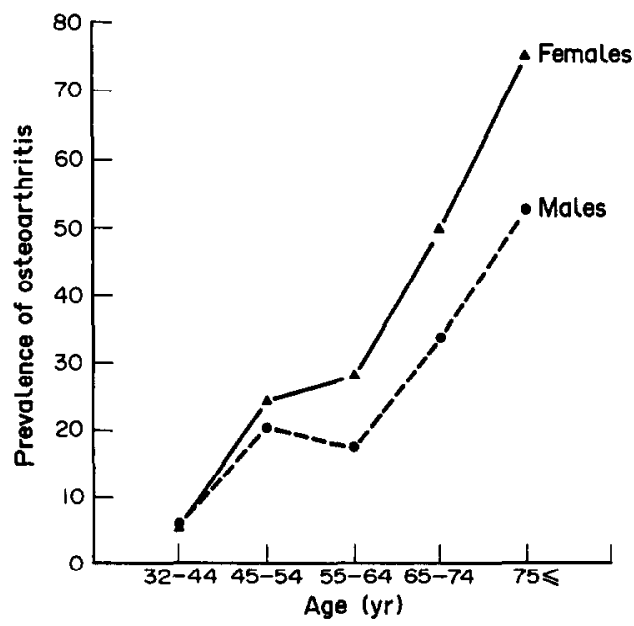

Fig. 1. Prevalence of ostenarthritis in at least one joint in the fingers and wrist. age as does the magnitude of the difference between the sexes. Among those subjects aged 75 years or older, the prevalence rates are $52.6 \%$ for males and $75.0 \%$ for females.

The number of joints with OA also increases with age for both males and females (Table 3). For example, for males the percentage of subjects with three or more joints with $\mathrm{OA}$ increases from $0.1 \%$ for those aged 44 years or younger to $15.6 \%$ for those aged 60 years or older $(p<0.001)$. The percentages for females are 1.0 and $28.2 \%$, respectively $(p<0.001)$. For the two older age categories, females have a greater

Table 3. Percentage of individuals with osteoarthritis in the finger and wrist joints, by age and sex categories, Tecumseh, Michigan, 1962-65

\begin{tabular}{ccrrrrr}
\hline & & \multicolumn{5}{c}{ No. of joints with OA } \\
\cline { 3 - 7 } & $n$ & 0 & $1-2$ & $3-5$ & $6-10$ & $11+$ \\
\hline Males & & & & & & \\
$\leqslant 44$ & 697 & 93.4 & 6.5 & 0.1 & 0.0 & 0.0 \\
$45-59$ & 494 & 82.0 & 14.4 & 2.8 & 0.6 & 0.2 \\
$60 \leqslant$ & 250 & 67.2 & 17.2 & 9.2 & 5.2 & 1.2 \\
Females & & & & & & \\
$\leqslant 44$ & 756 & 94.2 & 4.8 & 0.9 & 0.1 & 0.0 \\
$45-59$ & 572 & 75.2 & 14.9 & 6.4 & 3.0 & 0.6 \\
$60 \leqslant$ & 326 & 50.9 & 21.1 & 14.4 & 6.7 & 7.1 \\
\hline
\end{tabular}




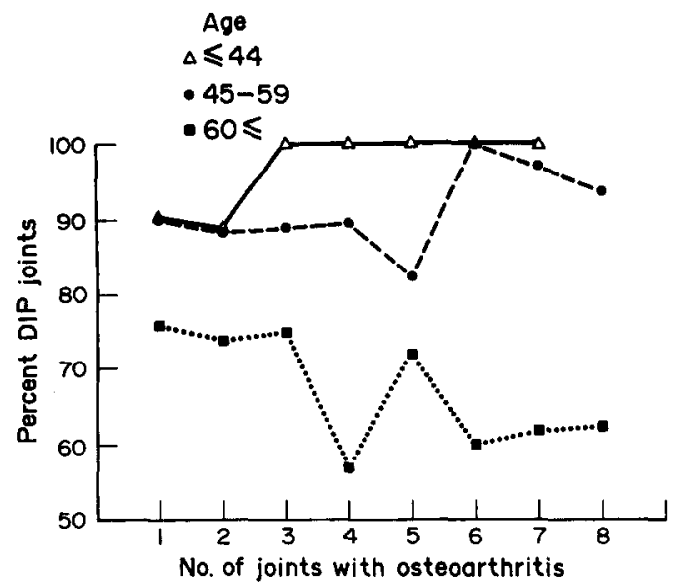

Fig. 2. Average percent of finger joints with osteoarthritis which are DIP joints.

number of affected joints than males $(p<0.001)$. It is noteworthy that $7.1 \%$ of women aged 60 or older had 11 or more affected joints compared to only $1.2 \%$ for men. For the youngest age category, the percentage of female subjects with OA $(5.8 \%)$ is slightly lower than that for males $(6.6 \%)$. However, those females with $O A$ have a slightly greater number of affected joints than do the males $(p=0.03)$. Of course, the number of subjects with $O A$ in the youngest age category is small so that the opportunity for general conclusions is limited.

Attention is now focused on the 24 joints of the eight fingers. Among those aged $45-59$ years with one to eight joints with $\mathrm{OA}$, on the average $90 \%$ of the joints with OA are DIP joints and $10 \%$ are PIP or MCP joints (Fig. 2). This is true even for individuals with six, seven or eight joints with OA. The average percentage for those aged 44 years or younger is even higher but not significantly different from the average percentage for those who are 45-59 years old. Thus, as is also shown in Table 2, OA in younger subjects is concentrated in the DIP joints and rarely is seen in the PIP or MCP joints. However, the average percentage for those aged 60 years or older is only $72 \%$, significantly lower than for the two younger age categories $(p<0.001)$ (Fig. 2). Thus, though $\mathrm{OA}$ is still concentrated in the DIP joints, the PIP and MCP joints are more likely to show signs of $O A$ in older than in younger subjects. Importantly, Fig. 2 shows that the pattern of the disease in the age groups differ. For example, consider two hypothetical individuals, one younger and one older than 60 years but both with six joints affected with OA. In the young individual, all six affected joints will be DIP joints while for the older individual only about three or four joints $(60 \%$ of $6=3.6)$ will typically be DIP joints (Fig. 2). This indicates that the increased prevalence of $\mathrm{OA}$ associated with the older age groups exhibits a different pattern among the joints than is observed among the younger individuals. In the younger subjects, OA is seen almost exclusively in the DIP joints and does not appear in the other joints until all the DIP joints have become involved. In the older subjects, OA is also concentrated in the DIP joints but the PIP and MCP joints exhibit the disease before all the DIP joints become involved.

The association between OA in the DIP and PIP joints also exhibits this age interaction (Table 4). The youngest age category is not shown since there was little disease in the PIP joints for these subjects. Among those aged 45-59 years with 1-2 DIP joints with OA, only $7.9 \%$ had at least one PIP joint with OA. For those with 6-8 DIP joints with OA, the percentage of subjects with at least one PIP joint with OA increases to $53.3 \%$. Thus, among younger subjects, the number of affected PIP joints increases with the number of affected DIP

Table 4. Percentages of individuals with OA in the DIP and PIP joints of the eight fingers, Tecumseh, Michigan, 1962-65

\begin{tabular}{|c|c|c|c|c|c|c|}
\hline & \multirow{2}{*}{$\begin{array}{c}\text { No. of DIP } \\
\text { joints with OA }\end{array}$} & \multirow[b]{2}{*}{$n$} & \multicolumn{4}{|c|}{ No. of PIP joints with OA } \\
\hline & & & 0 & $1-2$ & $3-5$ & $6-8$ \\
\hline \multicolumn{7}{|l|}{ Age 45-59 } \\
\hline & 0 & 826 & 98.5 & 1.2 & 0.2 & 0.0 \\
\hline & $1-2$ & 128 & 92.1 & 6.3 & 1.6 & 0.0 \\
\hline & $3-5$ & 37 & 91.8 & 8.1 & 0.0 & 0.0 \\
\hline & $6-8$ & 15 & 46.7 & 26.7 & 20.0 & 6.7 \\
\hline \multicolumn{7}{|l|}{ Age $60 \leqslant$} \\
\hline & 0 & 397 & 94.5 & 5.2 & 0.2 & 0.0 \\
\hline & $1-2$ & 108 & 75.9 & 15.7 & 8.3 & 0.0 \\
\hline & $3-5$ & 49 & 49.0 & 26.5 & 22.4 & 2.0 \\
\hline & $6-8$ & 24 & 20.8 & 25.0 & 20.8 & 33.3 \\
\hline
\end{tabular}


joints. Among those aged 60 years or older, the corresponding numbers are 24.1 and $79.2 \%$, also showing an increased involvement of the PIP joints with the number of affected DIP joints. The association between OA in the DIP and PIP joints is significant for both age categories $(p<0.001$ for each age category). In addition, the older subjects were more likely to have at least one PIP joint with OA than the younger subjects, for a given number of DIP joints with OA. For example, among those with 6-8 DIP joints with $\mathrm{OA}, 79.2 \%$ of the older subjects had at least one PIP joint with OA compared to only $53.3 \%$ for the younger subjects. Combining information across the four categories of number of DIP joints with OA, older subjects are at a significantly higher risk of having at least one PIP joint with $\mathrm{OA}(\mathrm{RR}=2.6,95 \% \mathrm{CI}=1.8$, 3.6).

The odds ratios for the association between $O A$ in the DIP and PIP joints of the same finger are presented in Table 5. These are Mantel-Haenszel odds ratios obtained by combining information from $2 \times 2$ tables constructed for each subject [16]. The $2 \times 2$ table for each subject had eight entries, one for each finger. Disease in the DIP joint of the finger defined the rows and disease in the PIP joint of the same finger defined the columns for each table. Information on the association between $\mathrm{OA}$ in the DIP and PIP joints was then combined across individuals. The odds ratio measures the magnitude of the association between $O A$ in the DIP and PIP joints of the same finger, controlling for the correlation among fingers from the same individual. The magnitude of the association is fairly constant across the four age and sex categories and the overall association ( $O R=1.24)$ is small, positive, but not statistically significant. The confidence interval is relatively narrow, ranging from 0.83 to 1.84 .

\section{DISCUSSION}

Because the radiologic diagnosis of OA is subject to individual interpretation, it is important that the reader of the X-rays be able to reproduce diagnoses as closely as possible. The primary reader for this project was shown to be as reliable as readers employed in this and other studies [see our Table 1 and Ref. 15]. Intrareader variability, however, will persist until the diagnostic criteria for the different levels of $\mathrm{OA}$ are more precisely stated and universally accepted.
Table 5. Odds ratios for the association between osteoarthritis in the DIP and PIP joints of the same finger, by age and sex categories, Tecumseh, Michigan, 1962-65

\begin{tabular}{lccc}
\hline & & Odds ratio & $\begin{array}{c}95 \% \text { confidence } \\
\text { interval }\end{array}$ \\
\hline Males & $45-59$ & 1.50 & $(0.29,7.87)$ \\
& $60 \leqslant$ & 1.37 & $(0.50,3.78)$ \\
Females & $45-59$ & 0.70 & $(0.23,2.11)$ \\
& $60 \leqslant$ & 1.28 & $(0.76,2.15)$ \\
Total & & 1.24 & $(0.83,1.84)$ \\
\hline
\end{tabular}

The pattern of $\mathrm{OA}$ in the finger and wrist joints of the adult participants of the Tecumseh Community Health Study in 1962-65 is similar to that reported by Acheson et al. [7] and Plato and Norris [4]. The disease is concentrated in the DIP joints of the fingers, and prevalence rates decreased centripetally along each finger. The DIP joint of the index finger has the highest prevalence rates for all age and sex categories, most likely due to the relatively high rate of involvement of this joint in many daily activities [6].

Though there is a positive association between OA in the DIP and PIP joints, there is only a weak association between OA in the DIP and PIP joints of the same finger $(O R=1.24$, $95 \% \mathrm{CI}=0.83,1.84)$. This is in contrast to Plato and Norris [4, p. 178] who suggested that "the presence of osteoarthritis in the distal joint may act ... as a predisposing factor enhancing the occurrence of the disease in the corresponding proximal joint."

Plato and Norris present an analysis based on pooled data which does not control for the lack of independence among fingers of the same individual. Their data show the relatively infrequent occurrence of OA of the DIP joints at early ages, the increasing number of affected joints with age, and the disproportionately high occurrence of OA in both PIP and DIP joints of the same finger at older ages. In the Tecumseh population, this same pattern was observed not only on the same finger but also when a DIP joint from one finger is compared to a PIP joint from another finger in the same individual. Thus, one can conclude only that OA in the DIP joints is associated with OA in the PIP joints, not that OA in the DIP joint of a specific finger is associated with OA in the corresponding PIP joint. In fact, controlling for the correlation among fingers of the same individual, the $O A$ status of a DIP joint is equally predictive of the $\mathrm{OA}$ status for the PIP joint of any finger and is 
not more highly predictive for its corresponding PIP joint.

A result of this analysis indicates an agedependent pattern for the presence of OA among the joints of the fingers. Among younger individuals, $\mathrm{OA}$ is observed almost exclusively among the DIP joints. However, among older individuals, OA was present in the PIP and MCP joints before all the DIP joints had exhibited disease and there was not a strong correlation for OA between DIP and PIP joints of the same fingers.

Such patterns might indicate different underlying etiologies at different ages. For example, the OA that appears at younger ages could be more strongly associated with factors specific to disease in the DIP joints. Possibly the appearance of $\mathrm{OA}$ in these joints is dominated by mechanical characteristics, such as stress measured by force/surface area of the joint. Disease that develops at the later ages in the DIP and PIP joints might be dominated by systemic factors to which all joints would be equally susceptible. The data upon which this report is based are cross-sectional and do not provide information about the time at which OA developed in specific joints. Incidence data are required to address this hypothesis more completely and current analyses of the Tecumseh incidence data may provide evidence for both systemic and mechanical etiologies.

\section{REFERENCES}

1. Kelsey JL. Epidemiology of Musculoskeletal Disorders. New York: Oxford University Press; 1982.

2. Kellgren JH, Lawrence JS. Osteo-arthritis and disk degeneration in an urban population. Ann Rheum Dis 1958; 17: 388-397.
3. National Center for Health Statistics. Prevalence of Osteoarthritis in adults by age, sex, race, and geographic area, United States 1960-1962. Vital and Health Statistics. PHS Pub. No. 1000-Series 11-No. 15. Public Health Service, Washington: U.S. Government Printing Office; 1966.

4. Plato $\mathrm{CC}$, Norris $\mathrm{AH}$. Osteoarthritis of the hand: age-specific joint-digit prevalence rates. Am J Epidemiol 1979; 109: 169-180.

5. Kellgren JH, Moore R. Generalized osteoarthritis and Heberden's nodes. Br Med J 1952; 1: 181-187.

6. Hadler NM, Gillings DB, Imbus HR, Levitin PM, Makuc D, Utsinger PD, Yount WJ, Slusser D, Moskevitz N. Hand structure and function in an industrial setting: influence of three patterns of stereotyped repetitive usage. Arth Rheum 1978; 21: 210-220.

7. Acheson RM, Chan Y, Clemett AR. New Haven survey of joint diseases XII: distribution and symptoms of osteoarthritis in the hands with reference to handedness. Ann Rheum Dis 1970; 29: 275-285.

8. Kuller LH: The epidemiology of osteoarthritis. In: Lawrence RC, Shulman LE, Eds. Epidemiology of Rheumatic Disease. New York: Gower Medical Publishing Ltd; 1984.

9. Acheson RM, Collart AB. New Haven survey of joint diseases XVII: relationship between some systemic characteristics and osteoarthrosis in a general population. Ann Rheum Dis 1975; 34: 379-387.

10. National Center for Health Statistics. Osteoarthritis and body measurements. Vital and Health Statistics. PHS Pub. No. 1000-Series 11--No 29. Public Health Service, Washington: U.S. Government Printing Office; 1968.

11. Dieppe P. Osteoarthritis: are we asking the wrong question? Br J Rheumatol 1984; 23: 161-165.

12. Mikkelsen WM, Duff IF, Dodge HJ. Age-sex specific prevalence of radiographic abnormalities of the hands, wrists, and cervical spine of adult residents of the Tecumseh, Michigan, Community health Study Area, 1962-1965. J Chron Dis 1970; 23: 151-159.

13. Napier JA. Field methods and response rates in the Tecumseh Community Health Study. Am J Publ Health 1962; 52: 208-216.

14. Atlas of Standard Radiographs of Arthritis. Philadelphia: FA Davis Co:; 1963

15. Wright EC, Acheson RM. New Haven survey of joint disease XI: observer variability in the assessment of x-rays for osteoarthrosis of the hands. Am J Epidemiol 1970; 91: 378-392.

16. Fleiss JL. Statistical Methods for Rates and Proportions. New York: Wiley; 1981. 2nd edn. 